\title{
Louis-Alexandre Bélisle au service du milieu des affaires au Québec
}

\section{Louis-Alexandre Bélisle and His Contribution to the Business Community of Québec Louis-Alexandre Bélisle al servicio de los negocios en Quebec}

\section{Josée Vincent}

Volume 56, numéro 4, octobre-décembre 2010

Parcours et trajectoires de médiateurs culturels

URI : https://id.erudit.org/iderudit/1029039ar

DOI : https://doi.org/10.7202/1029039ar

Aller au sommaire du numéro

Éditeur(s)

Association pour l'avancement des sciences et des techniques de la documentation (ASTED)

ISSN

0315-2340 (imprimé)

2291-8949 (numérique)

Découvrir la revue

Citer cet article

Vincent, J. (2010). Louis-Alexandre Bélisle au service du milieu des affaires au Québec. Documentation et bibliothèques, 56(4), 155-161.

https://doi.org/10.7202/1029039ar
Résumé de l'article

Si l'histoire a retenu l'œuvre de lexicographe de Louis-Alexandre Bélisle, auteur et éditeur du Dictionnaire de la langue française au Canada, il n'en est pas de même de son travail d'information et de vulgarisation portant sur le milieu des affaires au Québec. Dès 1932, Bélisle signe un premier ouvrage, Initiation pratique à la bourse, et conçoit le projet de publier une collection de manuels sur les affaires. Une première série paraîtra à l'enseigne des Éditions Les affaires qu'il dirige au début des années 1940, peu avant que soit créée en 1947, « La Bibliothèque de l’homme d'affaires ", chez « Bélisle, éditeur ». À travers ces publications, l'éditeur cherche non seulement à démystifier le monde des affaires, mais surtout à démontrer que le développement économique doit d'abord servir l'avancement de la nation.
Tous droits réservés (c) Association pour l'avancement des sciences et des techniques de la documentation (ASTED), 2010
Ce document est protégé par la loi sur le droit d'auteur. L’utilisation des services d’Érudit (y compris la reproduction) est assujettie à sa politique d'utilisation que vous pouvez consulter en ligne. 


\title{
Louis-Alexandre Bélisle au service du milieu des affaires au Québec ${ }^{\star}$
}

\author{
JOSÉE VINCENT \\ Co-directrice, Groupe de recherches et d'études sur le livre au Québec \\ Professeure, Université de Sherbrooke \\ josee.vincent@usherbrooke.ca
}

\begin{abstract}
RÉSUMÉ | ABSTRACTS | RESUMEN
Si l'histoire a retenu l'œuvre de lexicographe de Louis-Alexandre Bélisle, auteur et éditeur du Dictionnaire de la langue française au Canada, il n'en est pas de même de son travail d'information et de vulgarisation portant sur le milieu des affaires au Québec. Dès 1932, Bélisle signe un premier ouvrage, Initiation pratique à la bourse, et conçoit le projet de publier une collection de manuels sur les affaires. Une première série paraîtra à l'enseigne des Éditions Les affaires qu'il dirige au début des années 1940, peu avant que soit créée en 1947, "La Bibliothèque de l'homme d'affaires", chez "Bélisle, éditeur». À travers ces publications, l'éditeur cherche non seulement à démystifier le monde des affaires, mais surtout à démontrer que le développement économique doit d'abord servir l'avancement de la nation.
\end{abstract}

\section{Louis-Alexandre Bélisle and His Contribution to the Business Community of Québec ${ }^{* *}$}

If history has noted the work of the dictionary maker LouisAlexandre Bélisle, author and publisher of the Dictionnaire de la langue française $d u$ Canada, it has remained silent concerning his work conceiving and publishing business information for the layman. As early as 1932, he published Initiation pratique à la bourse and developed the idea of editing a series of business books, published by the Enseigne Les affaires that he managed in the early 1940 s shortly before the creation of La Bibliotheque de l'homme d'affaires by Bélisle, éditeur in 1947. The publisher used the series to shed light on the world of business but also to demonstrate that economic development must first and foremost serve the advancement of the nation.

Louis-Alexandre Bélisle al servicio de los negocios en Quebec ${ }^{* * *}$ La historia ha conservado la obra lexicográfica de Louis-Alexandre Bélisle, autor y editor del Dictionnaire de la langue française au Canada (Diccionario de la lengua francesa en Canadá); sin embargo, no ha sucedido lo mismo con su trabajo de información $y$ de vulgarización de los negocios de Québec. A partir de 1932, Bélisle publica una primera obra, Initiation practique à la bourse (Iniciación práctica en la bolsa), y decide publicar una colección de manuales sobre negocios. La primera edición se publica a través de la editorial Les Affaires, que dirige a comienzos de la década de los cuarenta. Poco tiempo después, en 1947, "La Bibliothèque de l'homme d'affaires" (La biblioteca del hombre de negocios) se crea en las instalaciones de Bélisle éditeur. Por medio de estas publicaciones, el editor no sólo busca desmitificar el mundo de los negocios, sino también demostrar que, ante todo, el desarrollo económico debe contribuir al progreso de la nación.
"En y mettant du "bon vouloir", nos relations deviendront plus cordiales, nous nous ferons aimer, estimer et admirer dans des ceuvres qui en valent la peine. Nous deviendrons pour les générations qui montent une source d'inspiration, des exemples dignes d'êtres suivis à cause de la considération qu'ils auront évoquée - et la race ne s'en portera que mieux parce qu'un pas de plus aura été franchi vers les sommets. Excelsior!"

Louis-Alexandre Bélisle, Les Affaires, vol xix, $\mathrm{n}^{\circ} 13$, février 1948, p. 8.

\section{L}

'EUVRE DE LOUIS-Alexandre BéLISLE (19021985) est gigantesque. On le sait, il est à la fois l'auteur, l'éditeur et l'imprimeur du Dictionnaire général de la langue française au Canada, ouvrage qui a marqué l'histoire de la langue au Québec et qui lui a valu entre autres choses d'être admis à la Société royale du Canada. L'histoire a néanmoins oublié plusieurs pans, sans doute moins prestigieux, de la carrière qu'il a menée dans le monde de l'imprimé. Tour à tour journaliste, auteur, traducteur, imprimeur et éditeur, Bélisle

* Les recherches qui ont été menées pour réaliser cet article s'inscrivent dans le cadre du projet du Dictionnaire des gens du livre au Québec, qui bénéficie d'une subvention du Conseil de recherche en sciences humaines du Canada (CRSH). Nous tenons à remercier Marie-Ève Riel, assistante de recherche au Groupe de recherche sur l'édition littéraire au Québec de l'Université de Sherbrooke, qui nous a fourni une aide très précieuse dans le dépouillement des publications de "Bélisle, éditeur ". La plupart de ces publications font partie des collections de Bibliothèque et Archives nationales du Québec. Nous avons également consulté le fonds Louis-Alexandre Bélisle ( $\mathrm{P}_{598}$ ), au Centre d'archives nationales de Québec, de Bibliothèque et Archives nationales du Québec.

* The research leading up to this article was undertaken for the Dictionnaire des gens du livre au Québec, and received a grant from the Social Sciences and Humanities Research Council of Canada (SSHRC). We would like to thank Marie-Ėve Riel, research assistant for the Groupe de recherché sur l'édition littérarie au Québec of the Université de Sherbrooke; she was very helpful in reading the publications of Bélisle, éditeur. Most of these publications are now part of the collections of the Bibliotheqque et Archives nationales du Québec. We also consulted the documents in the holdings of the Louis-Alexandre Bélisle $\left(\mathrm{P}_{598}\right)$ of the Centre d'archives nationales de Québec of the Bibliothèque et Archives nationales du Québec.

*** Las investigaciones que se han llevado a cabo para realizar este artículo se enmarcan dentro del proyecto Dictionnaire des gens du livre au Québec (Diccionario de los amantes del libro en Québec), que cuenta con una subvención del Consejo de investigación en ciencias humanas de Canadá (CRSH, por sus siglas en francés). Agradecemos a Marie-Ève Riel, investigadora adjunta del Grupo de investigación sobre edición literaria en Quebec, de la Universidad de Sherbrooke, quien nos ha prestado significativa ayuda en la clasificación de las publicaciones de Bélisle editéur. La mayoría de estas publicaciones forman parte de colecciones de Biblioteca y Archivos nacionales de Quebec. Asimismo, hemos consultado el fondo Louis-Alexandre Bélisle ( $\mathrm{P}_{598}$ ), en el Centro de archivos nacionales de Quebec, de la Biblioteca y Archivos nacionales de Quebec. 


\section{Tour à tour journaliste, auteur, traducteur, imprimeur et éditeur, Bélisle apparaît comme un véritable homme- orchestre dont le parcours présente, en condensé, une histoire complète des métiers du livre au Québec.}

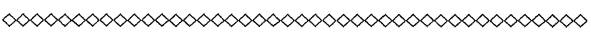

apparaît comme un véritable homme-orchestre dont le parcours présente, en condensé, une histoire complète des métiers du livre au Québec. Dans cet article, nous voulons nous arrêter à l'un des volets moins connus de ses activités, soit l'édition de manuels sur les affaires et le commerce.

Létude porte sur une vingtaine de titres que Bélisle publie à partir de 1936, parmi lesquels se retrouvent les treize ouvrages de "La Bibliothèque de l'homme d'affaires ». Ce qui a retenu notre attention, ce sont les objectifs qu'il se fixe. Alors que l'homme d'affaires cherche à exploiter un nouveau secteur du marché, celui des manuels conçus pour lenseignement professionnel, l'homme d'idées en profite pour faire œuvre de patriotisme; s'il faut bien apprendre à faire du commerce au Canada français, encore faut-il le faire non pas par intérêt personnel, mais pour le mieux-être de la nation !

Après avoir brièvement retracé le parcours éditorial de Louis-Alexandre Bélisle, nous rappellerons le contexte dans lequel ses publications sur le commerce et les affaires voient le jour. Nous aborderons ensuite le discours patriotique qui traverse ses ouvrages.

\section{Parcours d'un homme du livre}

Louis-Alexandre Bélisle est né en 1902 à St-Éloi de Témiscouata, un village situé près de Trois-Pistoles. Issu d'une famille de 13 enfants, il est le fils d'Hélène Rioux et de Georges Bélisle, fermier et meunier. Le jeune Louis-Alexandre est allé à lécole de rang, sans doute là où enseignait Mademoiselle Alice Michaud à qui il dédicacera le troisième ouvrage sur le commerce dont il est l'auteur, La vente au comptoir (Bélisle, 1942). Il fréquente ensuite le juvénat des Frères maristes de Lévis, où a enseigné son oncle, le frère Marie-Alexandre (Louis-Magloire Bélisle), dédicataire de son deuxième ouvrage, Crédits et recouvrements (Bélisle, 1940). Ce dernier aura une influence marquante sur son neveu, à qui il transmettra son amour de la langue et des origines du peuple canadiens-français. Louis-Alexandre Bélisle lui rendra hommage en lui dédicaçant également son Dictionnaire général de la langue française au Canada et en lui consacrant une longue notice dans ses Références biographiques (Bélisle, 1978).

En 1918, à lâge de 16 ans, Bélisle fait son entrée dans le monde du travail: instituteur, commis-comptable à la Banque canadienne nationale de St-Pacôme, puis comptable pour une compagnie forestière, la Power and Lumber, il poursuit en parallèle des cours par correspondance offerts par l'Université Queens, portant sur la pratique bancaire et commerciale. En 1927, il s'installe à Québec où il occupera pendant une dizaine d'années la charge déditorialiste des pages financières au journal $L e$ Soleil. Il enseigne également à l'École de commerce de l'Université Laval jusqu'au début des années 1940. Enseignement et pratique des affaires : voilà deux activités qui détermineront l'ensemble de son parcours éditorial.

Ses débuts au Soleil annoncent une longue carrière dans le monde de l'imprimé. En 1928, il fonde avec Raoul Renault la revue Les Affaires, qu'il dirigera jusqu'en 1963. Dès 1933, il rachète les parts de Renault et devient l'unique propriétaire de la revue. Trois ans plus tard, il acquiert l'imprimerie de La Semaine commerciale, hebdomadaire fondé en 1864, dont il ne se départira pas avant 1974. Il lance en 1937 Le Recueil, le premier digest français, qu'il vendra aux Jésuites dix ans plus tard. Enfin, il imprimera l'annuaire Marcotte de Québec et Lévis pendant quelque temps, à la fin des années 1940. La composition et l'impression de tous ces périodiques se font aux ateliers de La Semaine commerciale.

On le voit, Bélisle ne chôme pas. L'imprimeur profite d'ailleurs des temps morts à l'atelier pour produire des livres à compte d'auteur. Monographies de paroisse, livres d'histoire, pièces de théâtre, en tout, il publie une vingtaine de titres de 1936 à 1974 pour répondre à des demandes ponctuelles. Certains titres portent la mention "Bélisle, éditeur », d'autres l'achevé d'imprimer des ateliers de La Semaine commerciale seulement. Notons que lédition à compte d'auteur réserve parfois des surprises : c'est chez Bélisle que paraîtra en 1948 Les Plouffe, de Roger Lemelin. Sorti des presses en octobre, le roman sera réimprimé dès le mois suivant pour atteindre 10000 exemplaires. D'autres tirages seront produits par la suite. La publication des Plouffe demeure néanmoins une exception dans le parcours de Bélisle, puisque ce dernier évite autant que possible lédition littéraire pour concentrer ses activités dans le secteur scolaire. Un autre créneau qu'il explorera pendant la Guerre, à l'instar de bon nombre déditeurs, est celui de la réédition de classiques pour enfants. Conçue en collaboration avec la Librairie Beauchemin, la série « Les belles récompenses » sera produite en plusieurs formats à partir de 1940. Toutefois, Bélisle ne développera pas d'autres projets dans ce secteur à la suite de la reprise de lédition française, dans les années $1950^{1}$.

Lacquisition des presses de La Semaine commerciale permet à Bélisle d'échafauder un grand projet éditorial : la création de collections de manuels destinés à l'enseignement technique, alors en pleine expansion.

\footnotetext{
1. Au sujet de l'édition pendant la Seconde Guerre mondiale, voir Jacques Michon dir. 2004. Histoire de l'édition littéraire au Québec au XXe siècle, vol. 2 : Le temps des éditeurs. 1940-1959. Montréal : Fides. 533 p.
} 
L'idée, toute simple, consiste à rassembler des matériaux éprouvés, c'est-à-dire des manuels publiés en Europe et aux États-Unis dont il achète les droits, et des notes de cours produites par des professeurs avec lesquels il fonde, en 1942, la Société canadienne de technologie. Les membres de cette société, présidée par Bélisle, sont chargés de traduire et d'adapter les manuels à la réalité canadienne-française. Deux grands secteurs sont retenus: le commerce d'une part, comme on le verra plus loin, et les métiers de la construction et de l'industrie d'autre part.

Sous la bannière "Arts, métiers et technique" paraissent donc entre 1946 et 1952 les six titres de la collection "Les métiers de la construction", les sept volumes de "La Bibliothèque du machiniste " et "Les Manuels du mécanicien de machines fixes " en douze volumes. À la fin des années 1950, la série comprendra aussi deux ouvrages sur les voitures, un manuel de coiffure et quelques titres hors-collection ${ }^{2}$. D'abord vendus par souscription, puis en lot aux écoles et aux bibliothèques, voire à l'unité aux individus, les manuels techniques, plusieurs fois réimprimés et réédités pendant près de 30 ans, connaitront un extraordinaire succès. Après la cession de son entreprise aux Éditions Beauchemin en 1974, Bélisle continuera d'ailleurs d'en toucher les redevances jusqu'en 1981.

Les années 1950 voient la naissance de son grand œuvre, le Dictionnaire général de la langue française au Canada. Imprimé de 1955 à 1956 et vendu par livraison, louvrage est offert en 1957 aux souscripteurs et à la clientèle institutionnelle en format relié. Il connaîtra par la suite plusieurs réimpressions et rééditions. De nouveaux fascicules s'ajoutent à partir de 1958, pour former ce qu’on appellera le "Dictionnaire géant", comprenant outre le Dictionnaire de Bélisle, le Dictionnaire Oxford français-anglais, anglais-français, le répertoire des "Pays du monde ", une carte routière de la Province de Québec et plusieurs planches en couleur. Cette édition, que l'on peut acheter en format relié ou en fascicules (par abonnement ou dans les supermarchés), demeurera en circulation jusqu’à la fin des années 1960. Pressé d'actualiser son Dictionnaire à la suite de la création de l'Office de la langue française, Bélisle fait paraître une nouvelle édition en 1971, laquelle est réimprimée en 1974. Peu de temps auparavant, soit en 1969, il avait aussi lancé pour le marché scolaire le Petit dictionnaire canadien de la langue française, qui connaîtra lui aussi plusieurs éditions et réimpressions. Notons qu'une dernière édition du Dictionnaire sera produite par la Librairie Beauchemin en 1979. Faute de place, nous nélaborerons pas davantage l'histoire de cette formidable entreprise. Retenons

2. Pour qui s'intéresse à " Bélisle, éditeur ", l'établissement des collections peut devenir un véritable casse-tête. Dans les publicités et les listes d'ouvrages qu'il fait paraître, tant dans ses manuels que dans ses périodiques, Bélisle " triche " constamment, retirant et ajoutant des titres au gré des parutions, incluant à l'occasion certains titres dans deux collections. Mais les éditeurs ne sont pas là pour faciliter le travail des chercheurs.

\section{Dans la préface du Dictionnaire général de la langue française au Canada, Bélisle affirme que, dès les années 1930, il rêvait de publier une série d'ouvrages portant sur les pratiques commerciales.}

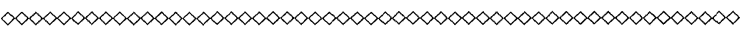

simplement que Bélisle y a consacré une grande partie de sa vie, l'idée de produire un Dictionnaire lui étant venue dès 1932, la collecte des mots et le travail de lexicographie sétant échelonnés des années 1930 aux années 1950, lédition, l'impression et la diffusion de l'œuvre dans tous ses formats ayant en quelque sorte meublé le reste de ses jours. Outre un siège à la Société royale du Canada, cette œuvre magistrale lui vaudra le Prix de la langue française de l'Académie française en 1958 et la Médaille d'or du Conseil de la Vie française en Amérique en 1971.

Au cours des années 1960, l'éditeur cherche à diversifier sa production. Il fonde un club du livre, la « Bibliothèque des grands auteurs ", une adaptation québécoise de la collection "Great Books of the Western World» de Mortimer Adler. Conçue pour la clientèle des collèges, la collection ne connaitra finalement pas beaucoup de succès. Ce type de production était-il trop éloigné de l'image de marque de la maison ? Les classiques étaientils toujours en demande alors que la réforme de lenseignement lancée à la suite du Rapport Parent encourageait plutôt la lecture d'œuvres québécoises ? Pour le moment, les raisons de cette mévente demeurent inconnues.

Les années 1970 vont entraîner la restructuration de l'entreprise. Bélisle arrive alors à lâge de la retraite. Déjà, en 1961, il avait formé une entité chargée uniquement de la composition au sein de son entreprise, Mono-lino enr., qu'il vendra à son fils Bernard en 1970. En 1974, il cède le fonds de "Bélisle, éditeur " aux Éditions Beauchemin, qui en assure la diffusion jusquen 1981. Bélisle séteint quatre ans plus tard, à lâge de 83 ans.

\section{L'imprimé au service des affaires}

Dans la préface du Dictionnaire général de la langue française au Canada, Bélisle affirme que, dès les années 1930, il rêvait de publier une série douvrages portant sur les pratiques commerciales. Professeur à l'École de commerce de l'Université Laval, il connaît bien les limites de la documentation dans cette discipline. Ainsi écrit-il dans son introduction à La vente au comptoir :

«Devant la volumineuse bibliographie de la vente à laquelle peuvent accéder nos concurrents de langue anglaise et notre indigence sous ce rapport, il est surprenant que nous ayons même pu faire figure de quelque chose à côté 


\section{Après la Guerre, le succès des manuels portant sur les métiers de la construction l'encourage à revoir ses plans.}

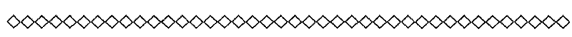

d'eux dans certains domaines de la sollicitation orale. [...]

$A u$ cours des dix prochaines années, nous devrons nous astreindre à évoluer vers les faits, les réalités, l'esprit scientifique, sous peine d'être irrévocablement assimilés par ceux qui, avant nous, ont saisi le sens et la portée du progrès matériel. Nous ne pouvons plus nous payer le luxe de vivre dans les nuages.

C'est dans cet esprit qu'ont été conçus et réalisés les ouvrages de la présente collection et plusieurs autres que nous nous proposons d'éditer.

[...]

On s'est longtemps demandé pourquoi si peu de nos établissements de détail prenaient la peine de donner à leur personnel des cours de vente. La réponse est simple: les manuels pouvant servir de base à ces cours manquaient totalement. Nous avons voulu combler ce vide [...]»

(Bélisle, $1947:$ 9-12)

Bélisle se met au travail et signe, en 1932, Initiation pratique à la bourse qui parait chez Albert Lévesque. Louvrage vise non seulement la clientèle universitaire, mais plus largement «les spéculateurs de langue française du Canada qui, jusqu'ici, ne possédaient aucun ouvrage de référence facile à comprendre et à consulter " (Bélisle, 193 : ix). L'auteur a mis cinq ans à rédiger ce manuel, puisant dans ses notes de cours et les éditoriaux qu'il signe pour Le Soleil, mais aussi dans des ouvrages dont il dresse la liste en bibliographie. À ce titre, il convient de souligner l'intégrité de l'homme qui cite systématiquement ses sources et n'hésite pas à rendre hommage à leurs auteurs.

Bien qu'il n'en soit nulle part fait mention, Initiation pratique à la bourse marque en quelque sorte le début d'une série. En 1936, Bélisle devenu imprimeur fait paraitre Expressions justes en affaires, d'Adjutor Fradet, à lenseigne des Éditions Les Affaires qu'il crée par la même occasion. Dans la préface - Bélisle signe souvent la préface des ouvrages qu'il publie, qu'il en soit ou non l'auteur -, il indique qu'il s'agit du deuxième volume d'une série qui devrait comporter plusieurs titres produits par des collaborateurs de la revue Les Affaires. Or, les titres annoncés s'avèrent être des manuscrits sur lesquels travaille Bélisle. Ce ne sera pas la première fois qu'un éditeur démarrera une collection en publiant ses propres ouvrages. "Charité bien ordonnée commence par soi-même » dit l'adage... À la suite de ce volume paraissent donc sous couvert de carton souple, orné d'un titre courant désignant leur appartenance à la nouvelle série "La Pratique des affaires", Crédits et recouvrements (1940), La vente au comptoir (1942) et Organisation et financement des entreprises (1947). Ces livres se vendent entre $1 \$$ et 1,60\$. Encore une fois, l'auteur a systématiquement repris des articles qu'il a rédigés pour Le Soleil, La Semaine commerciale et la revue Les Affaires, tout en s'inspirant d'ouvrages récents parus tant en anglais qu'en français.

Bélisle publie aussi hors-série d'autres ouvrages sur les affaires, qui paraissent avec la mention "Bélisle, éditeur ". Le premier, dont il est également l'auteur, connaîtra un succès phénoménal. Paru en 1942, Le Français des affaires sera réédité et réimprimé à maintes reprises3, la dernière édition connue datant de 1971. Louvrage se double d'un manuel à l'intention du maitre. Dans la préface, Bélisle affirme sềtre inspiré de Expressions justes en affaire qu'il a fait paraître huit ans plus tôt et dont le tirage est épuisé. Il ajoute souhaiter rééditer louvrage de Fradet, projet qui ne verra finalement jamais le jour4. Enfin, Bélisle publie en 1945 une conférence sur la bourse d'Antonin Lefebvre et un ouvrage paru d'abord en France et dont il acquiert les droits pour le Canada, $L a$ psychologie appliquée aux affaires, de Philippe Girardet, ancien directeur des ventes chez le fabricant de voitures Peugeot.

Après la Guerre, le succès des manuels portant sur les métiers de la construction l'encourage à revoir ses plans. À partir de 1947, il reprend les titres parus aux Éditions Les Affaires dans un nouveau format, celui de "La Bibliothèque de l'homme d'affaires" (Tableau 1). Recouverts d'une reliure en cuirette bleue avec titres dorés, ces petits ouvrages ( $5 \times 7$ pouces) présentent encore le titre courant "La Pratique des Affaires », mais c'est la mention «Bélisle, éditeur » qui s'affiche en page de titre, signe que la raison sociale de léditeur s'impose désormais, et pour de bon, aux yeux du public.

De 1947 à 1951, Bélisle réédite dans cette nouvelle collection Crédits et recouvrement et La psychologie appliquée aux affaires; il modifie Initiation pratique à la bourse, qui parait en 1948 sous le titre Marchés mobiliers et placements. En outre, il obtient les droits d'adaptation et de publication pour le Canada des quatre volumes du Traité de principes généraux de l'organisation de Georges de Leener, professeur à l'Université libre de Bruxelles, et des derniers volumes de l'Encyclopédie de la vente, de Philippe Girardet (le premier étant La psychologie appliquée aux affaires). Bélisle complète la série avec la réédi-

3. Nous en avons retracé sept, entre 1942 et 1971, mais il est fort probable que d'autres rééditions ou réimpressions nous aient échappées. Le manuel a aussi été vendu (et par conséquent, probablement réimprimé) pendant plusieurs années après 1971. Les chercheurs qui s'intéressent aux manuels scolaires savent combien il est souvent difficile d'avancer un nombre exact en la matière.

4. Nous ignorons la raison pour laquelle Bélisle n'a pas donné suite à ce projet. Certes, les deux ouvrages auraient fait double emploi. Dans les éditions subséquentes du Français des affaires, l'ouvrage de Fradet ne sera plus mentionné. 
tion de son Organisation et financement des entreprises, en $1956^{5}$.

De l'ensemble se dégage à la fois un aspect fonctionnel et une image de sérieux. Bélisle a bel et bien l'ambition de faire sa place dans le marché des manuels techniques. Toutefois, dès 1946, un concurrent redoutable apparaît: l'Office des cours par correspondance de l'Enseignement spécialisé, rattaché au ministère de l'Aide à la jeunesse. Au lieu de rivaliser avec l’organisme gouvernemental, Bélisle, en fin stratège, va plutôt chercher à s'en faire un allié. Il offre à ses dirigeants de planifier de concert leurs programmes éditoriaux pour éviter les dédoublements. La stratégie porte fruit, car les manuels de Bélisle seront utilisés dans les écoles techniques et dans les écoles de commerce, au même titre que ceux de l'Office. Grâce à cette entente, Bélisle parvient à maintenir une position de force dans ce secteur.

\section{Tableau 1}

Titres parus dans la collection « La Bibliothèque de l'homme d'affaires » chez « Bélisle, éditeur », de 1947 à 1956

\begin{tabular}{|c|c|}
\hline \multirow[t]{2}{*}{1947} & Crédits et recouvrement, L.-A. Bélisle \\
\hline & La psychologie appliquée aux affaires, P. Girardet \\
\hline 1948 & Marchés mobiliers et placements, L.-A. Bélisle \\
\hline \multirow[t]{5}{*}{1949} & Principes généraux de l'organisation, $\mathrm{G}$. de Leener \\
\hline & Organisation de l'emploi, G. de Leener \\
\hline & Organisation de la production, G. de Leener \\
\hline & Organisation financière et administrative, G. de \\
\hline & Leener \\
\hline \multirow[t]{4}{*}{1950} & Sélection et formation des vendeurs, P. Girardet \\
\hline & Prospection de la clientèle, P. Girardet \\
\hline & Service des ventes, P. Girardet \\
\hline & La vente et la publicité, P. Girardet \\
\hline 1956 & $\begin{array}{l}\text { Organisation et financement des entreprises, L.-A. } \\
\text { Bélisle }\end{array}$ \\
\hline
\end{tabular}

«La Bibliothèque de l'homme d'affaires"se vend 36 \$ en 1953 ( 3 \$ l'unité), un prix qui sera maintenu dans les années 1960. Bélisle multiplie les efforts pour garder les prix bas, voire les abaisser. Par exemple, après sêtre doté d'un atelier de reliure au début des années 1940, il négocie l'achat de matériaux à moindre coût, offrant à l'entrepreneur de Chicago qui avait relié Crédits et recouvrement en 1940 d'acheter ses encres usagées. Surtout, il sollicite systématiquement le gouvernement, l'empressant d'inclure son entreprise dans la liste du patronage, tant pour loctroi de contrats d'impression que pour lachat de manuels par les institutions.

5. Un dernier ouvrage, Techniques modernes de supervision, d'un certain Lateiner, semble avoir été ajouté vers 1975, mais nous n'avons pas encore pu en faire la vérification, livre en main.
On peut affirmer que les manuels de commerce connaitront autant de succès que les manuels de métiers, du moins si l'on se fie, faute d'informations précises sur les tirages, au nombre élevé de réimpressions.



Pour faire connaître ses publications, Bélisle fait flèche de tout bois. Ne reculant pas devant l'autopromotion, il inclut des publicités dans ses manuels et dans ses périodiques et fait paraître des recensions dans les pages de La Semaine commerciale et de la revue Les Affaires. Un long article très élogieux, signé par le colonel J.-E. Marquis, conservateur de la Bibliothèque de la législature, paraît ainsi dans la livraison de février 1948 des Affaires (Bélisle, 1948 : 17-21). Bélisle sollicite également d'autres périodiques, notamment l'Almanach Beauchemin dont il profite de limmense rayonnement. Pour la distribution, il s'entend avec la Librairie Beauchemin, mais aussi avec des distributeurs tels Grolier, en 1951, ou la Sondec, en 1967. Enfin, il n'hésite pas à écrire directement aux directeurs décoles et de bibliothèques, aux libraires-grossistes, aux députés et aux hautsfonctionnaires. La correspondance regorge de lettres priant les uns et les autres d'acheter ses manuels, un geste patriotique, argumente-t-il. En effet, nous verrons bientôt en quoi ces manuels véhiculent un discours éminemment nationaliste.

On peut affirmer que les manuels de commerce connaîtront autant de succès que les manuels de métiers, du moins si lon se fie, faute d'informations précises sur les tirages, au nombre élevé de réimpressions (deux à quatre par titre). On rééditera d'ailleurs la série complète dans les années 1960, au moment où l'atelier passe à l'impression offset. En parallèle, d'autres manuels de bureautique et de comptabilité seront aussi produits hors collections.

\section{S'enrichir, certes, mais pour la nation !}

$\mathrm{Au}$ moment où Bélisle enseigne à l'École de commerce, l'Occident traverse une grave crise économique à la suite du crash boursier de 1929. Les années 1930 sont particulièrement difficiles alors que sévit le chômage au sein de la classe ouvrière, très populeuse au Canada français, comme lillustre si bien Les Plouffe de Roger Lemelin. Le travail de Bélisle vise donc, dans un premier temps, à reconstruire l'image du milieu des affaires et à montrer comment l'on peut investir intelligemment. Ainsi affirmera-t-il en 1932, dans son Initiation à la bourse : 


\section{Bélisle considère que le pouvoir de changer les choses est entre les mains des individus et non pas entre celles de l'État ou des associations.}

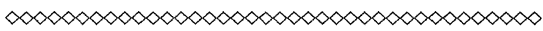

"Ceux qui mettent le plus en doute l'utilité des Bourses sont presque invariablement des gens qui y ont englouti de l'argent en spéculant imprudemment ou sans posséder les connaissances voulues. Ou bien, ils confondent avec des opérations permises sur ce marché des pratiques malhonnêtes perpétrées en dehors de la Bourse. En spéculation comme ailleurs il faut distinguer entre la compétence et l'incompétence [...] "

(Bélisle, 1932: 8)

Notons que Bélisle est un homme d'affaires prudent, qui préfère les vertus de la modération. S'il importe de développer léconomie, encore faut-il le faire sans chercher à s'enrichir à n'importe quel prix. C'est avant tout cette idée de mesure et d'organisation qu'il souhaite transmettre :

«En préparant ce travail, je me suis efforcé d'en faire un instrument utile, pratique et de consultation facile. Mon plus sincère désir est de contribuer à l'avancement économique de mes compatriotes en dirigeant leurs efforts vers les grandes artères de l'organisation et non sur les sentiers incertains des innovations dont le temps n'a pas encore consacré la valeur»

(Bélisle, 1952 : x)

On le voit, derrière la mission professorale transparaît la leçon de civisme. Pour l'enseignant, l'apprentissage du commerce, qui bénéficie d'abord à l'individu, doit ultimement servir la collectivité.

"Comme tous les autres grands marchés mobiliers, du reste, la Bourse de Montréal a, si l'on peut s'exprimer ainsi, le sens de ses responsabilités envers le pays et le peuple canadien. Ceux qui placent des capitaux dans les valeurs inscrites sur notre bourse ont foi dans le pays, dans ses perspectives d'avenir [...]»

(Bélisle, $1932: 8$ )

Ainsi, la participation au progrès social demeure une préoccupation constante chez Bélisle. "Se rendre utile ", "rendre service " et "souci de perfection " sont autant d'expressions qui reviennent constamment sous sa plume. Bélisle considère que le pouvoir de changer les choses est entre les mains des individus et non pas entre celles de l'État ou des associations. Ainsi prône-t-il la prise en charge individuelle qui se traduit par l'inves- tissement dans les entreprises locales, seules garantes de lévolution sociale. Dans un article intitulé «Le potentiel humain et notre expansion économique » (Bélisle, 1944 : 48-51), il écrit :

"Certes, on est en affaires pour faire de l'argent, mais pas rien que de l'argent. La base du succès - même pour réaliser beaucoup d'argent - est avant tout de se rendre utile, de rendre des services que personne n'a encore rendus, ou de les rendre d'une façon plus parfaite que ceux qui s'en occupent déjà »

(Bélisle, 1944a : 51).

Cette insistance trouve sa raison dêtre dans le contexte économique des années 1930 et 1940. Peut-être faut-il rappeler que le Québec connaît pendant la guerre un développement industriel accéléré, ce qui ne manque pas de plaire à Bélisle, mais que ce développement est porté par des intérêts canadiens-anglais, voire américains, ce qui, à l'inverse, l'agace profondément. Non pas qu'il entretienne l'antagonisme à l'égard de la "race » anglo-saxonne. Au contraire, il voue une admiration sans bornes aux Américains, fiers bâtisseurs d'une civilisation fortement industrialisée qu'il érige en modèle de bonheur et de vertu. Ainsi écrit-il, au retour d'un séjour en Nouvelle-Angleterre :

"Ici et là une ferme, des chalets, des plaines marécageuses; partout des routes merveilleuses, des chemins de fer, des résidences d'un enviable confort et d'une beauté qui ne souffre que de très rares exceptions. [...]

Partout règne l'ordre, la bonne humeur et une aisance modeste, mais visible et généralisée "

(Bélisle, 1944b : 10-11).

À lécoute d'une société en pleine transformation, son discours progressiste s'oppose à certains tenants d'un nationalisme conservateur américanophobe. En réalité, ce qui l'agace, c'est l'immobilisme, voire le défaitisme des Canadiens français.

"C'est Louis Hémon qui a dit "Au pays de Québec, rien ne doit changer!" Un moment de réflexion nous permettra de détruire cette affirmation dont les touristes peuvent se rassasier, mais non les hommes d'affaires. [...]

Ce marché est à nous. Si d'autres s'en sont emparés c'est parce que, le plus souvent, nous négligeons d'en tirer parti, c'est que nous n'en connaissons pas les possibilités et que nous nous abstenons de prendre notre part"

(Bélisle, 1944a : 48-50).

«Ramassons nos énergie et osons!» (Bélisle, 1944c:52), écrira-t-il encore, de sa plume enflammée ! 
"L'audace et l'initiative qu'exigent les affaires sont le prolongement normal et un raffinement des qualités qu'ont dîu employer les pionniers. Nous sommes au carrefour d'une transition, dont l'effet aura sur notre groupe ethnique des conséquences dont trop peu semblent saisir toutes les implications et l'ultime aboutissement. Aiguillons nos énergies du bon côté et OSONS »

(Bélisle, 1944c : 54 ; c'est l'auteur qui souligne).

Nous pourrions multiplier à l'infini les citations appuyant ce discours du progrès, prônant lavancement du peuple canadiens-français dans le concert des nations. Elles signalent, entre autres, lobjectif poursuivi à travers la publication des manuels, soit celui de doter les Canadiens français d'outils leur permettant de maîtriser leur économie et, ultimement, leur destin.

En lançant des collections de manuels sur le commerce, Bélisle court certains risques. Certes, il est extrêmement bien placé pour le faire. Professeur à l'École de commerce, directeur de périodiques dont il noircit volontiers lui-même les pages, il connaît parfaitement les besoins, le réseau et la clientèle. Imprimeur et éditeur, il sait également mesurer ce que représente une telle aventure. Reste que ce créneau éditorial est totalement nouveau au moment où il s'y lance. En ce sens pouvonsnous affirmer qu'il prêche par l'exemple, n'hésitant pas à foncer, à faire preuve de cet esprit d'initiative qu'il réclame de ses contemporains. L'histoire lui donnera raison, comme le prouve le succès de ses manuels plusieurs fois réédités pendant près de quarante ans.

Bélisle atteint donc l'objectif qu'il sétait fixé au début des années 1930, soit de doter les écoles techniques en manuels adaptés à la réalité canadienne-française. Mais laction de Bélisle déborde largement des cadres de l'édition et de l'enseignement. Le discours nationaliste qu'il véhicule à travers ses publications vise clairement à éveiller le patriotisme chez tous les individus rattachés au monde des affaires, qu'il s'agisse de simple commis ou de patrons d'entreprises influents. Éditeur engagé, Bélisle contribue ainsi à démystifier le monde du commerce, à une époque où cet univers demeure largement dominé par les Canadiens anglais et les Américains. Plus encore, il offre à ses concitoyens les moyens de se dépasser.

Reconnu d’abord à cause de son amour de la langue qui lui a fait produire le tout premier dictionnaire québécois, Louis-Alexandre Bélisle est un personnage fascinant qui mérite une place à part dans l'histoire du livre au Québec. Rares sont les individus qui, comme lui, aient non seulement touché à toutes les fonctions du milieu du livre, mais qui, à maints égards soient parvenus à y exceller. (-)

\section{Éditeur engagé, Bélisle contribue ainsi à démystifier le monde du commerce, à une époque où cet univers demeure largement dominé par les Canadiens anglais et les Américains.}

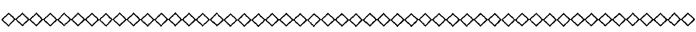

\section{Sources consultées}

Bélisle, Louis-Alexandre. 1932. Initiation pratique à la bourse. Montréal : Éditions Albert Lévesque. Coll. « Action canadienne-française ». xv, $387 \mathrm{p}$.

Bélisle, Louis-Alexandre. 1940. Crédits et recouvrements. Québec: Éditions Les Affaires. xv, $230 \mathrm{p}$.

Bélisle, Louis-Alexandre. 1942. La vente au comptoir. Québec: Éditions Les Affaires. $220 \mathrm{p}$.

Bélisle, Louis Alexandre. 1944a. Le potentiel humain et notre expansion économique. Les Affaires vol. xvi no 9 (octobre) : 48-51.

Bélisle, Louis Alexandre. 1944b. L'industrialisation, facteur d'influence politique. Les Affaires vol. xvi n ${ }^{\circ} 10$ (novembre) : 9-12.

Bélisle, Louis Alexandre. 1944c. Ramassons nos énergies et osons. Les Affaires vol. xvi ${ }^{\circ} 11$ (décembre) : 52-54.

Bélisle, Louis Alexandre. 1948. Le succès attend les hommes de bonne volonté. Les Affaires vol. xix n ${ }^{\circ} 13$ (février) : 6-8.

Bélisle, Louis-Alexandre. 1952 (première édition parue en 1947). Introduction. In Organisation et financement des entreprises. Québec : Bélisle, éditeur. xiv, 288 p.

Bélisle, Louis-Alexandre. 1978. Références biographiques. CanadaQuébec, vol. 1: A-Ca. Montréal : Éditions de la famille canadienne limitée. $116 \mathrm{p}$.

Lemelin, Roger. 1948. Les Plouffe. Québec : Bélisle, éditeur. 470 p. 